Article

\title{
Preparation and Characterization of Insoluble Silk Fibroin/Chitosan Blend Films
}

\author{
Mariana Agostini de Moraes, Grinia Michelle Nogueira, Raquel Farias Weska and \\ Marisa Masumi Beppu *
}

School of Chemical Engineering, University of Campinas, UNICAMP, 13083-970, Campinas, SP, Brazil; E-Mails: marimoraes@feq.unicamp.br (M.A.M.); grinia.nogueira@ gmail.com (G.M.N.); weska@feq.unicamp.br (R.F.W.)

* Author to whom correspondence should be addressed; E-Mail: beppu@ feq.unicamp.br; Tel.: +55-19-3521-3893; Fax: +55-19-3521-3922.

Received: 25 October 2010; in revised form: 26 November 2010 / Accepted: 2 December 2010 / Published: 13 December 2010

\begin{abstract}
The aim of this study was to prepare and characterize membranes of silk fibroin (SF) and chitosan (CHI) blends. Moreover, a conformation transition of SF to a more stable form induced by the addition of CHI was verified. Blend membranes were prepared, after $\mathrm{pH}$ adjustment, in different ratios, and physical integrity, crystallinity, structural conformation and thermal stability were characterized. The results of crystallographic analysis (XRD) indicated the tendency to higher structural organization caused by the addition of CHI. Fourier transformed infrared spectroscopy (FTIR) showed that SF is present in a more stable form in the presence of a CHI content of only $25 \mathrm{wt} \%$. Thermal analysis indicated that SF is thermally stable and that when its proportion in the blend increases, the temperature at which degradation is initiated also increases.
\end{abstract}

Keywords: biopolymers; structural conformation; blends

\section{Introduction}

Silk fibroin (SF) is a natural fibrous protein spun from Bombyx mori silkworm. The cocoon of the silkworm is mainly composed of sericin and fibroin. Sericin is a glue-like protein that holds SF fibers together in the cocoon case. SF is composed of a repetitive sequence of amino acids: glycine, alanine 
and serine, and - as all fibrous proteins - is not soluble in water due to its high concentration of hydrophobic amino acids [1].

The molecular conformation of SF membranes is an important parameter that needs to be controlled, since it affects their physical and chemical properties. SF has two types of molecular conformation of the secondary structure, called silk I and silk II. Silk I is a metastable form of SF that is soluble in water and non-crystalline; random coil and $\alpha$-helix conformations are usually called silk I. On the other hand, silk II is a highly stable and organized structure that is insoluble in water; the $\beta$-sheet conformation is called silk II [2]. Generally, both silk I and silk II are present in SF products, but it is their relative proportions that will define the final properties.

SF solution does not present enough intermolecular hydrogen bonds to stabilize its structure. To promote the transition of SF conformation from silk I to silk II, some researchers propose physical and chemical treatments using high temperature, high humidity and immersion in organic solvents [3-5]. However, with these treatments, SF films become brittle in the dry state and would be unsuitable for practical use [6]. If the dry state is required and the brittleness is undesirable, SF properties can be improved by blending with other natural polymers [7-10].

Chitosan (CHI) is a natural polymer obtained from the deacetylation of chitin, a major component of crustacean shells. CHI has active sites capable of interacting with SF macromolecules, which is a necessary condition for the compatibility of polymers in solutions and films [11].

Blending natural polymers is sometimes a challenge because natural polymers do not have regular molecular structures. They have a wide range of molecular masses and are highly influenced by processing factors that can result in different products. Due to these factors, controlling the processing conditions are key parameters in achieving reproducible results.

Some authors have already studied blends of SF and CHI and verified a structural transition of SF from silk I to silk II in the presence of CHI content up to $40 \mathrm{wt} \%$ [3,7,12]. However, all these authors have performed post treatments on the blends in order to induce a more stable and crystalline structure.

The goal of this study was to prepare and characterize SF/CHI blend films, aiming at an insoluble film, with a more stable and crystalline structure. The films were characterized by X-ray diffraction (XRD), Fourier transformed infrared spectroscopy with attenuated total reflection device (FTIR-ATR), differential scanning calorimetry (DSC) and thermogravimetry (TGA), to evaluate their chemical properties and thermal stability.

\section{Experimental Section}

Cocoons of Bombyx mori silkworm were supplied by Bratac (São Paulo, Brazil). The preparation of SF aqueous solution was accomplished by the method proposed by Nogueira et al. [13], with modifications. Briefly, the cocoons were degummed three times with 1 wt $\% \mathrm{Na}_{2} \mathrm{CO}_{3}$ solution at $85{ }^{\circ} \mathrm{C}$ to remove sericin. The $\mathrm{SF}$ fibers were dried and dissolved in a ternary solvent of $\mathrm{CaCl}_{2}: \mathrm{CH}_{3} \mathrm{CH}_{2} \mathrm{OH}: \mathrm{H}_{2} \mathrm{O}$, in a molar ratio of $1: 2: 8$, at $85{ }^{\circ} \mathrm{C}$, to a SF salt solution of $5 \mathrm{wt} \%$. The SF salt solution was then dialyzed against distilled water for 3 days, at $10{ }^{\circ} \mathrm{C}$, with water changes every 24 hours. The final aqueous SF solution was diluted with distilled water to a $1 \mathrm{wt} \% \mathrm{SF}$ solution concentration. 
Chitosan (Sigma, minimum deacetylation degree of 85\%) was dissolved in a 2 vol\% acetic acid solution to a final CHI solution concentration of $1 \mathrm{wt} \%$. The CHI flakes were dispersed with a mechanical stirrer for 4 days, until complete dissolution. The final CHI solution was vacuum filtered to remove any undesirable impurities.

\subsection{Blend Preparation}

The solutions of SF and CHI were mixed for 15 minutes with stirring at room temperature in several SF:CHI mixing ratios (100:0, 75:25, 50:50, 25:75 and 0:100). The blend films were prepared by casting the mixed solutions onto polystyrene plates and allowing the solvent to evaporate at room temperature. In this study, the films were not treated or neutralized in order to evaluate the solubility of the membranes in their non-treated state.

\subsection{Measurements}

The solubility of the blend films was qualitatively estimated by immersion of the films in $50 \mathrm{~mL}$ of distilled water for 24 hours, at room temperature. After the incubation, the blended films were evaluated regarding their physical integrity, as qualitatively soluble or non-soluble in water.

XRD diffractograms were obtained using a X'PERT PW3050 (PHILIPS) diffractometer, and CuK $\alpha$ radiation with a wavelength of $1.54 \AA$ was used. The scanning speed was $0.6 \% \mathrm{~min}$, and the measurement range was $2 \theta=10-35^{\circ}$. XRD analysis was conducted to study changes in the crystallinity of the blended films.

FTIR-ATR spectra were obtained using an Illuminate R2 spectrometer in the spectral region of 650 to $4,000 \mathrm{~cm}^{-1}$, to investigate the changes induced by CHI to the secondary structure of SF films.

DSC measurements were performed with a DSC-50 (Shimadzu), in the temperature range of $25-500{ }^{\circ} \mathrm{C}$ and with a heating rate of $10{ }^{\circ} \mathrm{C} / \mathrm{min}$. TGA was performed in a TGA-50 (Shimadzu) in the temperature range of $25-600{ }^{\circ} \mathrm{C}$ with a ramp rate of $10^{\circ} \mathrm{C} / \mathrm{min}$ and a $\mathrm{N}_{2}$ flow of $50 \mathrm{~mL} / \mathrm{min}$.

\section{Results and Discussion}

To prepare the blends, some parameters needed to be adjusted to obtain a homogeneously mixed solution and a film without macroscopic phase separation.

$\mathrm{SF}$ and CHI solutions underwent phase separation when mixed in their as-prepared form. This fact can be explained if we consider the $\mathrm{pH}$ values of the as-prepared solutions. SF aqueous solution naturally has a $\mathrm{pH}$ of 7 , while $\mathrm{CHI}$ solution (dissolved in acetic acid) has a $\mathrm{pH}$ value of ca. 4 . In environments above $\mathrm{pH} 6.5, \mathrm{CHI}$ precipitates because it is near the isoelectric $\mathrm{pH}$, where it has the same quantity of negative and positive charges [14]. On the other hand, when $\mathrm{pH}$ values are below 5 , SF aqueous solution undergoes gelation, forming an irreversible hydrogel, with a highly organized molecular structure [15]. In this way, it is necessary to adjust the $\mathrm{pH}$ values of SF and CHI solutions prior to mixing them to avoid phase separation and to allow more effective interactions between them. In this study, there was the need to adjust the $\mathrm{pH}$ values of the $\mathrm{SF}$ and $\mathrm{CHI}$ solutions to $c a$. 5.5. Under this condition, $\mathrm{CHI}$ presents its amino groups in a protonated form, which allows more interactions with the carboxyl groups of SF. 


\subsection{Physical Integrity}

The solubility of the blended films in water was evaluated and the results are shown in Table 1. It is possible to observe that, except for the pure SF film, the blends were not soluble in water and their physical structure was maintained after immersion in water. This result can be an indication of the formation of a more stable SF structure when blended with CHI. SF films without physical or chemical treatments are highly soluble in water, as shown in Table 1, due to the lack of intermolecular hydrogen bonds to stabilize the structure. It is possible to suppose that $\mathrm{CHI}$ interacts with SF chains and some hydrogen bonds are formed, avoiding the solvation of SF in water.

In addition, the influence of the acetic acid (CHI solvent) on the stabilization of SF films shall also be considered. Prior to the blend formation, the $\mathrm{pH}$ of $\mathrm{CHI}$ solution was adjusted to 5.5, which could have partially neutralized the acetic acid. However, the remaining acetic acid could also play a role in inducing a structural change to the SF chains. It is known that SF presents a more stable structure in the presence of ethanol and methanol. Kweon and co-authors [16] verified that the acetic acid present in CHI solution could play the same role as the organic solvents in inducing a more stable conformation in SF chains. The authors attributed the silk II structure of SF in the blend to the acetic acid used as the solvent for $\mathrm{CHI}$ rather than the interaction with $\mathrm{CHI}$ itself.

Table 1. Results of physical integrity test of the SF/CHI blends.

\begin{tabular}{cc}
\hline SF:CHI & Physical Integrity \\
\hline $100: 0$ & Soluble \\
$75: 25$ & Non soluble \\
$50: 50$ & Non soluble \\
$25: 75$ & Non soluble \\
$0: 100$ & Non soluble \\
\hline
\end{tabular}

\section{1. $X R D$}

Figure 1 presents the X-ray diffractograms of the SF/CHI blended films. The pure SF film (Figure 1a) presented an XRD pattern typical of amorphous substances, with the absence of crystallinity peaks, characterizing a silk I secondary structure. This result was expected as no treatments were performed to increase the crystallinity of the films.

By adding $\mathrm{CHI}$ into the blends (Figure 1(b-d), some crystalline halos at $2 \theta=14^{\circ}$ and $17^{\circ}$ could be observed. The intensity of these halos is proportional to the increase of CHI content. These halos are attributed to a silk II structure; highly crystalline [17]. The change in the crystallinity of the blend films is possibly due to interactions between SF and CHI chains that can interact by hydrogen bonding, thus stabilizing the SF molecular structure [18]. Another halo can be observed at $2 \theta=20^{\circ}$, characteristic of CHI diffractograms. This halo presents different intensities in the blend's diffractograms, according to CHI content [16]. 
Figure 1. XRD of SF/CHI blended films with the ratio SF:CHI of (a) 100:0, (b) 75:25, (c) 50:50, (d) 25:75, (e) 0:100.

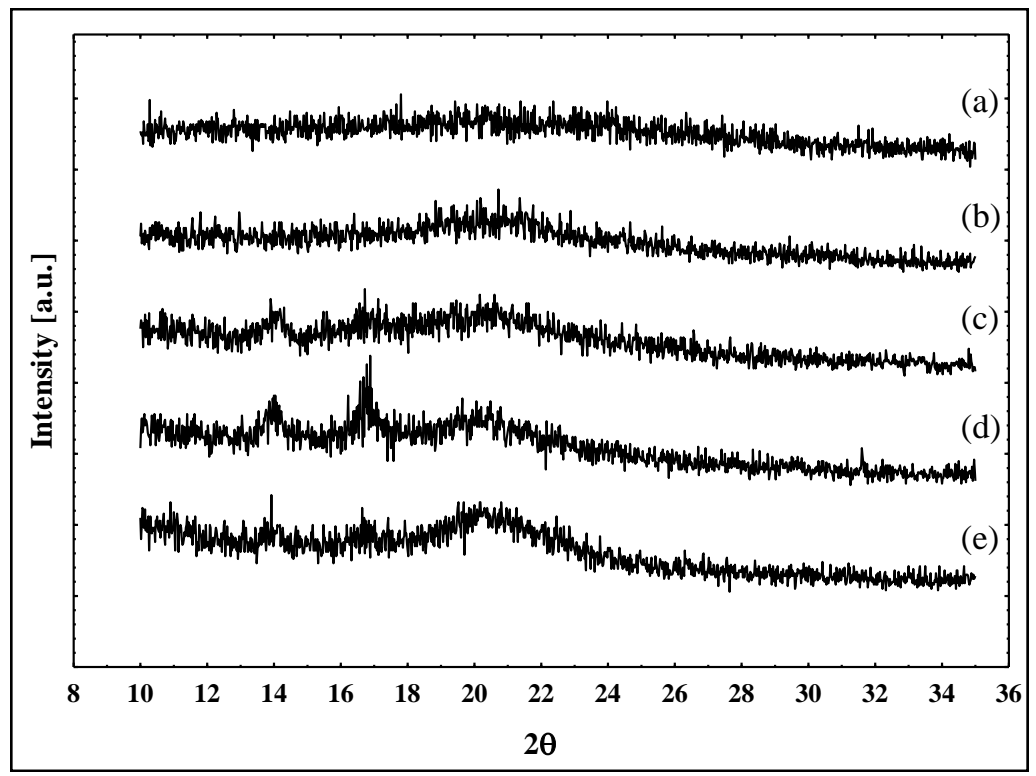

\subsection{FTIR-ATR}

By analyzing the infrared spectrum, the structural conformation of SF can be determined, depending on the wavenumber location of the absorption bands of amides I, II and III. The infrared spectra of the SF and CHI blends are shown in Figure 2.

Pure SF film [Figure 2(a)] presented absorption bands at $1,641 \mathrm{~cm}^{-1}$ and $1,632 \mathrm{~cm}^{-1}$ (amide I) and $1,515 \mathrm{~cm}^{-1}$ (amide II), corresponding to the SF silk II structural conformation ( $\beta$-sheet). Other absorption bands were observed at $1,530 \mathrm{~cm}^{-1}$ (amide II) and $1,237 \mathrm{~cm}^{-1}$ (amide III), which are characteristic of the silk I conformation (random coil and $\alpha$-helix) [19]. This indicates that silk I and silk II structures are presented simultaneously in pure SF films; however, the high solubility of this film (see Section 3.1) indicates the predominance of the silk I structure.

For pure CHI films [Figure 2(e)], some characteristic absorption bands were also observed. The absorption band at $1,646 \mathrm{~cm}^{-1}$ is attributed to the $\mathrm{C}=\mathrm{O}$ bond and the band at $1,584 \mathrm{~cm}^{-1}$ to $-\mathrm{NH}_{2}$ stretching. The absorption band at $1,150 \mathrm{~cm}^{-1}$ is related to the anti-symmetric stretching of C-O-C bridge, and the bands at $1,059 \mathrm{~cm}^{-1}$ and $1,026 \mathrm{~cm}^{-1}$ to skeletal vibrations involving the $\mathrm{C}-\mathrm{O}$ stretching [16]. By diminishing CHI content in the blend films, the intensity of the absorption bands corresponding to CHI C-O stretching (1,059 and 1,026 $\left.\mathrm{cm}^{-1}\right)$ also decreased.

The infrared spectrum of the blend containing $75 \mathrm{wt} \%$ of SF [Figure 2(b)] presented more defined absorption bands of amides I and II, attributed to the silk II conformation. The absorption bands related to silk I structure in the pure SF spectra [Figure 2(a)] were no longer observed, except for the band corresponding to amide III $\left(1,233 \mathrm{~cm}^{-1}\right)$. In the infrared spectra of the other blend films [Figure 2(c,d)], absorption bands related to silk I and II could be observed. However, in these blends, a tendency to present a less stable structure was revealed: silk I structure was more present when compared to the spectra shown in Figure 2(b). In addition, changes in SF amide groups and the disappearance of $\mathrm{C}=\mathrm{O}$ and $\mathrm{NH}_{2}$ groups of $\mathrm{CHI}$ in all the studied blends indicate that these groups may have participated in specific interactions between $\mathrm{SF}$ and $\mathrm{CHI}$. 
Figure 2. FTIR-ATR spectra of SF/CHI blended films with the ratio SF:CHI of (a) 100:0, (b) 75:25, (c) 50:50, (d) 25:75, (e) 0:100.

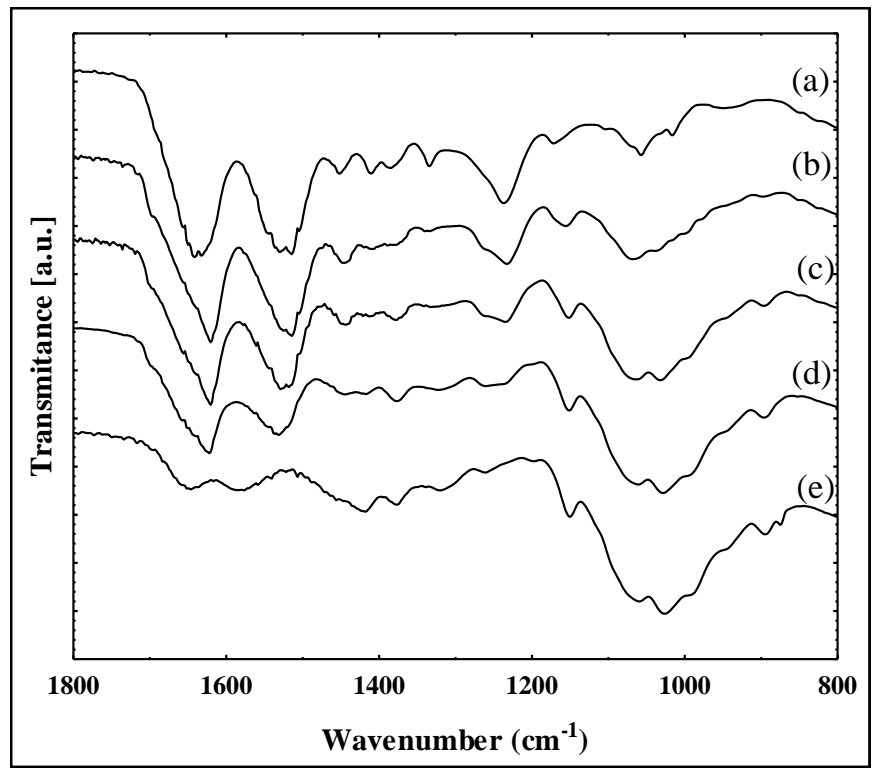

Therefore, the addition of $\mathrm{CHI}$ in these blends caused structural conformation modifications to SF. Once the silk I and silk II conformations are present in the films, we can indicate a possible conformational transition by analyzing the absorption bands. We observed that these structural modification were most pronounced in the blend film SF:CHI 75:25, demonstrating that there is a maximum $\mathrm{CHI}$ content that should be added to SF solution in order to induce a structural conformation modification. Other research groups also studied blends of SF and CHI and verified an increase in stability and crystallinity with a maximum CHI content of $40 \mathrm{wt} \%[7,12,16]$.

\subsection{Thermal Analysis}

Figure 3 shows the DSC thermograms for the SF/CHI blends. Endothermic peaks below $150{ }^{\circ} \mathrm{C}$ were observed, related to the evaporation of the adsorbed water in the films, in all analyzed blends.

Pure SF film, Figure 3a, presented an endothermic peak related to its thermal degradation at $283{ }^{\circ} \mathrm{C}$. Thermal degradation peaks of SF films at temperatures below $290{ }^{\circ} \mathrm{C}$ are characteristic of amorphous SF (silk I), and are present when SF films are not submitted to any kind of physical or chemical treatment to induce its conformation to a more stable structure (silk II) [20]. Thus, this result is in accordance with our SF film when no treatments were employed. In addition, an endothermic peak at $204.6{ }^{\circ} \mathrm{C}$ and an exothermic peak at $224{ }^{\circ} \mathrm{C}$ were observed (in the scale shown in Figure 3, these peaks are slightly observed). The endothermic peak is attributed to the molecular motion within the $\alpha$-helix crystals and the exothermic peak is attributed to the crystallization during heating from a silk I to a silk II structure $[11,16]$.

The thermograms of the blend films, Figure 3(b-d), presented a mixture of components. The peaks at 204.6 and $224{ }^{\circ} \mathrm{C}$ were no longer observed in the blend films. A similar behavior was observed by Kweon and co-authors [16] in A. pernyi SF/CHI blends, where the typical exothermic peak due to the crystallization of SF chains was not presented in the blends. This indicates that the acetic acid used to dissolve $\mathrm{CHI}$ diffuses in the blend film and induced a precrystallization of SF chains. 
Pure CHI film [Figure 3(e)] presented a characteristic exothermic peak at $294{ }^{\circ} \mathrm{C}$. This peak is attributed to a complex process including dehydration of the saccharide rings, depolymerization and decomposition of the acetylated and deacetylated units of polymer [16].

Figure 3. DSC thermograms of SF/CHI blended films with the ratio SF:CHI of (a) 100:0, (b) 75:25, (c) 50:50, (d) 25:75, (e) 0:100.

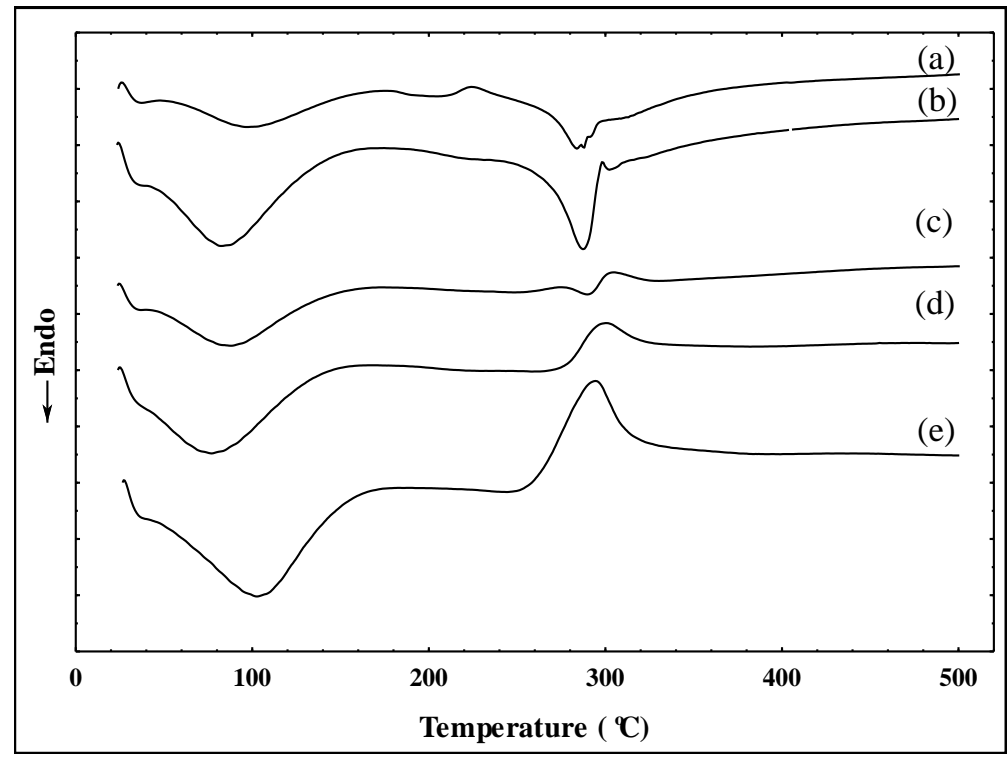

Thermogravimetric curves of the blend films are shown in Figure 4. The initial weight loss, below $100{ }^{\circ} \mathrm{C}$, was due to water evaporation. Increasing the SF content in the blends increased the temperature of thermal degradation, indicating that SF provided a thermal stability in the blended films.

Figure 4. Thermogravimetric curves of SF/CHI blended films with the indicated SF:CHI composition.

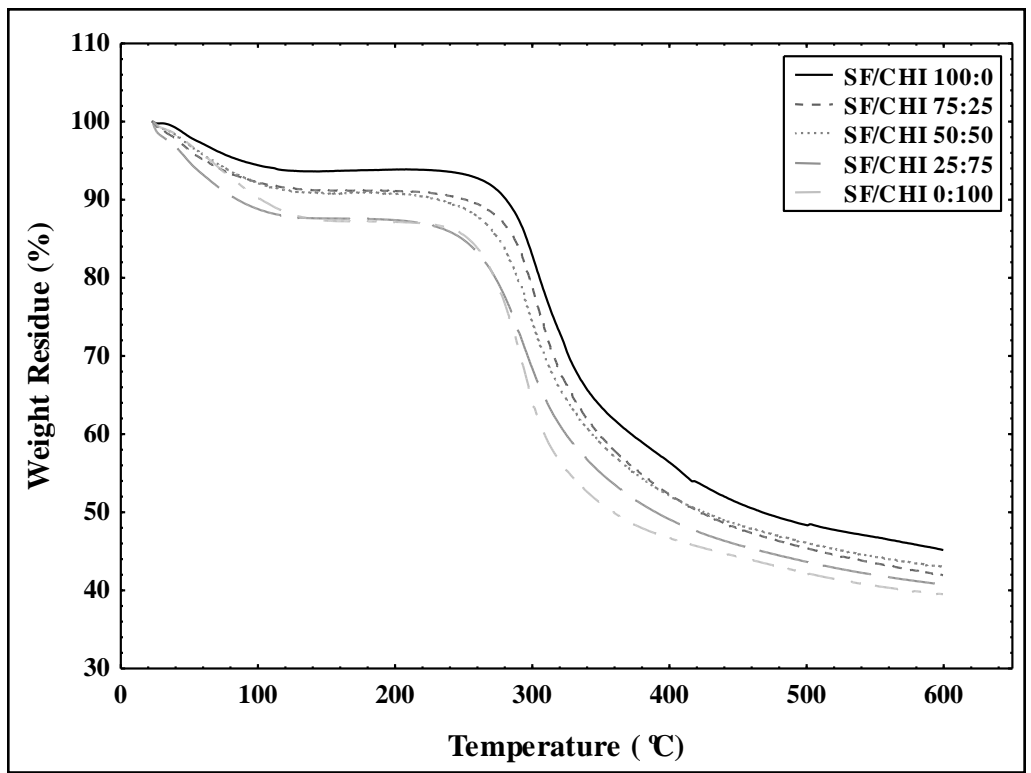


Figure 5 presents the weight residues and differential weight residue curves for the blend film of $\mathrm{SF} / \mathrm{CHI}$ 50:50. All other blend films presented similar behavior, with a weight loss peak below $100{ }^{\circ} \mathrm{C}$ (water loss) and peak of thermal degradation near $300{ }^{\circ} \mathrm{C}$. It was observed that the increase of SF content increased the temperature of thermal degradation at $c a .4{ }^{\circ} \mathrm{C}$ for each blend.

Figure 5. Thermogravimetric curve and differential weight residue curve of SF/CHI 50:50 blended film.

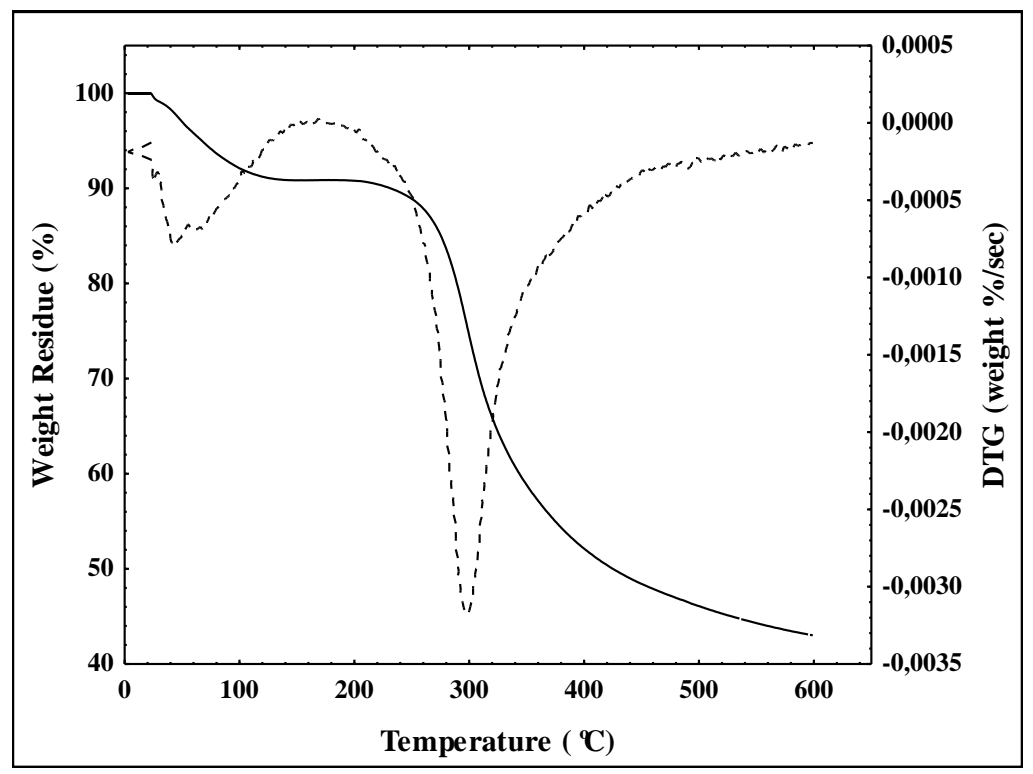

\section{Conclusions}

SF/CHI blended films were prepared after necessary $\mathrm{pH}$ adjustment were made to the SF and CHI solutions, to allow the formation of homogeneous blend films without macroscopic phase separation.

Characterization of the blends showed that silk I and silk II structures exist simultaneously in the films, however, when $25 \mathrm{wt} \%$ of $\mathrm{CHI}$ is present in the blend, the most stable and crystalline structure is obtained. In addition, blend films with higher SF content are more resistant to thermal degradation.

\section{Acknowledgements}

The authors thank CNPq for financial support.

\section{References}

1. Altman, G.H.; Diaz, F.; Jakuba, C.; Calabro, T.; Horan, R.L.; Chen, J.; Lu, H.; Richmond, J.; Kaplan, D.L. Silk-based biomaterials. Biomaterials 2003, 24, 401-416.

2. Motta, A.; Fambri, L.; Migliaresi, C. Regenerated silk fibroin films: Thermal and dynamic mechanical analysis. Macromolecul. Chem. Phys. 2002, 203, 1658-1665.

3. Chen, X.; Li, W.; Yu, T. Conformation transition of silk fibroin induced by blending chitosan. J. Polym. Sci. Part B Polym. Phys. 1997, 35, 2293-2296.

4. Kawahara, Y.; Furukawa, K.; Yamamoto, T. Self-expansion behavior of silk fibroin film. Macromolecul. Mater. Eng. 2006, 291, 458-462. 
5. Putthanarat, S.; Zarkoob, S.; Magoshi, J.; Chen, J.A.; Eby, R.K.; Stone, M.; Adams, W.W. Effect of processing temperature on the morphology of silk membranes. Polymer 2002, 43, 3405-3413.

6. Li, M.; Lu, S.; Wu, Z.; Tan, K.; Minoura, N.; Kuga, S. Structure and properties of silk fibroin-poly(vinyl alcohol) gel. Int. J. Biol. Macromolecul. 2002, 30, 89-94.

7. Kweon, H.; Ha, H.C.; Um, I.C.; Park, Y.H. Physical properties of silk fibroin/chitosan blend films. J. Appl. Polym. Sci. 2001, 80, 928-934.

8. Lee, K.G.; Kweon, H.Y.; Yeo, J.-H.; Woo, S.-O.; Lee, J.-H.; Park, Y.H. Structural and physical properties of silk fibroin/alginate blend sponges. J. Appl. Polym. Sci. 2004, 93, 2174-2179.

9. Vasconcelos, A.; Freddi, G.; Cavaco-Paulo, A. Biodegradable materials based on silk fibroin and keratin. Biomacromolecules 2008, 9, 1299-1305.

10. Marsano, E.; Canetti, M.; Conio, G.; Corsini, P.; Freddi, G. Fibers based on cellulose-silk fibroin blend. J. Appl. Polym. Sci. 2007, 104, 2187-2196.

11. Sashina, E.S.; Janowska, G.; Zaborski, M.; Vnuchkin, A.V. Compatibility of fibroin/chitosan and fibroin/cellulose blends studied by thermal analysis. J. Therm. Anal. Calorimet. 2007, 89, 887-891.

12. Park, S.J.; Lee, K.Y.; Ha, W.S.; Park, S.Y. Structural changes and their effect on mechanical properties of silk fibroin/chitosan blends. J. Appl. Polym. Sci. 1999, 74, 2571-2575.

13. Nogueira, G.M.; Rodas, A.C.D.; Leite, C.A.P.; Giles, C.; Higa, O.Z.; Polakiewicz, B.; Beppu, M.M. Preparation and characterization of ethanol-treated silk fibroin dense membranes for biomaterials application using waste silk fibers as raw material. Bioresour. Techn. 2010, 101, 8446-8451.

14. Suh, J.-K.F.; Matthew, H.W.T. Application of chitosan-based polysaccharide biomaterials in cartilage tissue engineering: A review. Biomaterials 2000, 21, 2589-2598.

15. Kim, U.-J.; Park, J.; Li, C.; Jin, H.-J.; Valluzzi, R.; Kaplan, D.L. Structure and properties of silk hydrogels. Biomacromolecules 2004, 5, 786-794.

16. Kweon, H.Y.; Um, I.C.; Park, Y.H. Structural and thermal characteristics of Antheraea pernyi silk fibroin/chitosan blend film. Polymer 2001, 42, 6651-6656.

17. Tao, W.; Li, M.; Zhao, C. Structure and properties of regenerated Antheraea pernyi silk fibroin in aqueous solution. Int. J. Biol. Macromolecul. 2007, 40, 472-478.

18. She, Z.; Zhang, B.; Jin, C.; Feng, Q.; Xu, Y. Preparation and in vitro degradation of porous three-dimensional silk fibroin/chitosan scaffold. Polym. Degrad. Stabil. 2008, 93, 1316-1322.

19. Rusa, C.C.; Bridges, C.; Ha, S.-W.; Tonelli, A.E. Conformational changes induced in Bombyx mori silk fibroin by cyclodextrin inclusion complexation. Macromolecules 2005, 38, 5640-5646.

20. Freddi, G.; Pessina, G.; Tsukada, M. Swelling and dissolution of silk fibroin (Bombyx mori) in N-methyl morpholine N-oxide. Int. J. Biol. Macromolecul. 1999, 24, 251-263.

(C) 2010 by the authors; licensee MDPI, Basel, Switzerland. This article is an open access article distributed under the terms and conditions of the Creative Commons Attribution license (http://creativecommons.org/licenses/by/3.0/). 